\title{
Habitat fragmentation and biodiversity collapse in neutral communities
}

\author{
Ricard V. Solé $^{\mathrm{a}, \mathrm{b}, *}$, David Alonso ${ }^{\mathrm{a}, \mathrm{c}}$, Joan Saldaña ${ }^{\mathrm{d}}$ \\ ${ }^{a}$ ICREA-Complex Systems Lab, Universitat Pompeu Fabra, Dr Aiguader 8008003 Barcelona, Spain \\ b Santa Fe Institute, 1399 Hyde Park Road, New Mexico 87501, USA \\ c Department of Ecology, Facultat d'Ecologia, Diagonal, Barcelona, Spain \\ d Departament d'Informàtica i Matemàtica Aplicada, Universitat de Girona, 17071 Girona, Spain
}

Received 3 November 2003; received in revised form 5 December 2003; accepted 7 December 2003

\begin{abstract}
Models of habitat fragmentation have mainly explored the effects on a few-species ecologies or on a hierarchical community of competitors. These models reveal that, under different conditions, ecosystem response can involve sharp changes when some given thresholds are reached. However, perturbations, recruitment limitation and other causes may prevent competitive hierarchies from actually operating in natural conditions: the process of competitive exclusion underlying hierarchies could not be a determinant factor structuring communities. Here we explore both spatially-implicit and spatially-explicit metapopulation models for a competitive community, where the colonization-extinction dynamics takes place through neutral interactions. Here species interactions are not hierarchical at all but are somehow ecologically equivalent and just compete for space and resources through recruitment limitation. Our analysis shows the existence of a common destruction threshold for all species: whenever habitat loss reaches certain value a sudden biodiversity collapse takes place. Furthermore, the model is able to reproduce species-rank distributions and its spatially explicit counterpart predicts also species-area laws obtained from recent studies on rainforest plots. It is also discussed the relevance of percolation thresholds in limiting diversity once the landscape is broken into many patches. (c) 2004 Elsevier B.V. All rights reserved.
\end{abstract}

Keywords: Habitat fragmentation; Biodiversity collapse; Neutral communities

\section{Introduction}

When native vegetation is cleared (usually for agriculture or other kinds of intensive exploitation) habitats which were once continuous become divided into separate fragments (Hanski, 1999; Pimm, 1991). After intensive clearing, the separate fragments tend to

\footnotetext{
* Corresponding author.

E-mail address: ricard.sole@upf.edu (R.V. Solé).
}

be very small islands isolated from each other by crop land and pasture. Fragmentation and loss of habitat are recognized as the greatest existing threat to biodiversity. Fragmentation is occurring, to various degrees, in nearly all of the major habitat types found throughout the world. Rainforests, which comprise $6 \%$ of the world's land area and which contain at least $50 \%$ of the world's total species, are being cleared and fragmented at a rate far exceeding all other types of habitat. Human-caused habitat fragmentation precipitates 
biodiversity decline because it destroys species, disrupts community interactions, and interrupts evolutionary processes (Ehrlich and Ehrlich, 1981; Pimm et al., 1998; Leakey and Lewin, 1995; Levin, 1999).

How does habitat fragmentation affect biodiversity in rich ecosystems? The answer to this question will depend on the type of interactions displayed by the species under consideration. Previous multispecies models of habitat fragmentation have assumed the presence of a hierarchical organization of competitive interactions. The best known example is Tilman's model, defined as (Tilman, 1994; Tilman et al., 1994; Tilman and Kareiva, 1997; Kareiva and Wennengren, 1995):

$$
\frac{\mathrm{d} p_{i}}{\mathrm{~d} t}=c_{i} p_{i}\left(1-D-\sum_{j=1}^{i} p_{j}\right)-m_{i} p_{i}-\sum_{j=1}^{i-1} c_{j} p_{i} p_{j}
$$

where $p_{i}(i=1, \ldots, n)$ is the fraction of the occupied habitat for the $i$-th competitor and $D$ the fraction of destroyed patches. Species specific colonization and extinction rates are represented by $c_{i}$ and $m_{i}$, respectively. The single (non-zero) equilibrium state for this system is given by $\mathbf{p}^{*}=\left(p_{1}^{*}, \ldots, p_{n}^{*}\right)$, where

$$
\begin{array}{r}
p_{i}^{*}=1-D-\frac{m_{i}}{c_{i}}-\sum_{j=1}^{i-1}\left(1+\frac{c_{j}}{c_{i}}\right) p_{j}^{*}, \\
i=1, \ldots, n
\end{array}
$$

Here there is a hierarchical order from the first (best) competitor to the last (worst) one. Extinction is assumed to be constant for all species and the colonization rates are chosen as $c_{i}=m /(1-q)^{2 i-1}$, where $q$ the abundance of the best competitor and $i$ is the order label in the competitive hierarchy. This choice assumes a trade-off: the best competitor $(i=1)$ is the worst colonizer. Furthermore, such a choice allows to recover a geometric distribution of species-abundances. One of the most important consequences of this model is the presence of a debt: a cascade of extinctions that occur generations after fragmentation (Tilman et al., 1994).

Further analysis by Lewi Stone expanded the previous approach to coral reefs (Stone, 1995). By using some studies on coral reefs in Israel, where the best colonizer has the largest abundance, he correlated mortality rates with colonization, thus assuming the presence of a trade-off. Using this approximation, Stone derived a simple relation between the number of extinct species and fragmentation: $E=\sqrt{D / \alpha}$ where $\alpha$ is the ratio between the abundance of two successive species used to set the initial distribution of abundances. Stone's work reveals that very small amounts of habitat destruction have a huge detrimental effect on coral reefs: a destruction of only $10 \%$ of the habitat can trigger the loss of up to half the total species. From Tilman's et al. work, the effects on a forest ecosystem would be less important, and the same amount of destruction would only remove $5 \%$ of the species.

The competition-colonization trade-off seems to be fundamental among competing species: the ability to persist on a site, i.e., to be a good competitor, versus the ability to get to a new site, i.e. to be a good colonizer. As a mechanism of coexistence was termed fugitive coexistence (Fisher et al., 1951; Skellam, 1951). Since these two pioneering studies this trade-off has been explored in several forms (MacArthur and Wilson, 1967; Tilman, 1982, 1994; Stone, 1995; Crowley and McLetchie, 2002)—see also Hanski (1999) and reference therein. But, to what extent are actually hierarchies structuring natural competitive communities?

It is important to remark here that the existence of competitive hierarchies has been clearly shown to structure competitive communities under experimental conditions. When there is an only limiting resource, poorer competitors are displaced by superior competitors as the resource is depleted (Tilman, 1982). However, some recent studies point to the evidence that competitive hierarchies are not always at work under natural conditions. Rather than competitive local exclusion under a strict hierarchy, studies on tree diversity in neotropical forests seem to indicate that recruitment limitation appears to be a major source of control of tree diversity (Hubbell et al., 1999; Tilman, 1999). In other words, the winners of local competition are not necessarily the best (local) competitors but those that happened to colonize that particular site first. Such priority effects have been reported also in other systems (Barkai and McQuaid, 1988).

Furthermore, a community of ecologically equivalent species rather than a hierarchically structured competitive community is the key assumption of a theory supported by extensive field research mainly in rainforest that, as E.O. Wilson says, "could be one 
of the most important contributions to ecology of the past half century" (Hubbell, 2001). Thus, in this paper, instead of considering a hierarchy of competitors, we use a metapopulation model where species are ecologically equivalent in their colonization ability. In a metapopulation context such an equivalence regarding the fraction of colonized habitat and extinction thresholds is achieved by an equal colonization-to-extinction ratio $\left(c_{i} / m_{i}\right)$ (Hanski, 1999). We are considering a community where species may just colonize empty sites and share the same $c_{i} / m_{i}$ ratio. This constancy can be seen as another possible expression of the fundamental competition-colonization trade-off. The better colonizer a species is, the worst ability to persist on a site (high extinction rate) it must have. Our model is as general as that of Tilman (1994), but instead of assuming a strict competitive hierarchy it assumes strict neutral competitive interactions.

Given that neutrality has been shown to account for important regularities of community structure, such as species relative abundance distributions and species richness, and given also that it has been tested in very different natural ecosystems (Hubbell, 2001), the conclusions, in particular, with regard to the consequences of habitat loss, drawn from our approach might even be more realistic and valid that those drawn form previous work.

In the second part of the paper, we expand the model into a spatially-explicit context. Interestingly, the spatially-explicit model supports the spatially-implicit derived results and is able to produce realistic speciesabundance and species-area curves (SAR). In the spatially-explicit model species are located on a squared lattice and colonize just empty neighboring sites. Better colonizers have greater extinction rates and tend to persist less on a site. There is a fair amount of evidence from many plant communities for a trade-off between competitive strength, i.e, the ability to persist, and dispersal, i.e, colonization ability (Lehman and Tilman, 1997). In forests limited by light, the trees with the greatest ability to tolerate shade are often competitively superior but severely limited by dispersal. Dispersal limitation may be critical to understanding species coexistence and community dynamics in tropical forests. It has been observed that some tree species exhibits densely aggregated spatial distributions independent of habitat structure. Nearly all species exhibiting pronounced aggregation had seeds dispersed by gravity or by ballistic dispersal. Thus, interactions between dispersal and the ability to persist can affect spatial structure in populations in ways that could alter local diversity and competitive interactions at the community level. Such a scenario is known to provide an essentially unlimited diversity.

\section{Mean field model}

A spatially-implicit, mean field (MF) metapopulation model of such recruitment limitation process can be easily formulated by means of a generalization of Levins model (Levins, 1969) as:

$$
\frac{\mathrm{d} p_{i}}{\mathrm{~d} t}=f_{i}(\boldsymbol{p})=c_{i} p_{i}\left(1-D-\sum_{j=1}^{S} p_{j}\right)-\phi\left(c_{i}\right) p_{i},
$$

including habitat fragmentation. Here the extinction rate $m_{i}$ has been replaced (last term) by $m_{i}=\phi\left(c_{i}\right)$ which gives the functional form of the trade-off. This trade-off is chosen to rend all species ecologically equivalent in their colonization ability, where as in Hanski (1999), this equivalent ability means the same ability to colonize and persist if they were in isolation. So, we assume the same $c / m$ ratio for all species. Equivalently, we can write the functional form of the trade-off then as:

$\phi(c)=\alpha c$

We assume $\alpha<1$, which means that no species would go to extinction in isolation. Therefore, such trade-off implies that high (low) colonization rates are linked to high (low) local extinction rates, and can be seen as a particular expression of the fundamental competition-colonization (persistence ability versus colonization ability) trade-off (Lehman and Tilman, 1997).

As a result of this choice, it is easy to see that the total fraction of occupied patches $P=\sum_{i} p_{i}$ will evolve in time as:

$\frac{\mathrm{d} P}{\mathrm{~d} t}=<c>(1-D-P-\alpha) P$

where $\langle c\rangle=\sum_{i} p_{i}(t) c_{i} / P(t)$ is the average colonization rate at time $t$, and it is used that the average 
extinction rate is $\langle\phi(c)\rangle=\alpha<c\rangle$ under the assumption of a linear trade-off given by (4). Hence, Eq. (5) has a unique positive stationary value, namely, $P^{*}=1-D-\alpha$, which is globally asymptotically stable for any initial condition $P(0)>0$. This implies that the main result of this mean-field model (where spatial correlations are not taken into account) is that the global population will experience a collapse at a critical threshold of habitat destruction $D_{\mathrm{c}}=1-\alpha$ since, for this value of $D, P^{*}=0$. And thus a diversity collapse will occur at $D_{\mathrm{c}}$, instead that at increasing values for $D$, as it was shown by Tilman et al. (1994).

Eq. (3), endowed with a positive initial condition $\boldsymbol{p}^{0}=\left(p_{1}^{0}, \ldots, p_{S}^{0}\right)$, has as a unique stable equilibrium $\boldsymbol{p}^{*}$ which is given by

$$
\begin{array}{r}
p_{i}^{*}=p_{i}^{0} \exp \left(c_{i} \int_{0}^{\infty}(1-D-\alpha-P(t)) \mathrm{d} t\right), \\
i=1, \ldots, S
\end{array}
$$

with $P(t)$ a solution of (5) with initial condition $P^{0}=$ $\sum_{i} p_{i}^{0}$. However, such an equilibrium is not asymptotically stable. Notice that, for any $D<D_{\mathrm{c}}$, any initial condition $\boldsymbol{p}^{0}$ with $P^{0}=1-D-\alpha$ is also an equilibrium of the model since, in this case, $P(t)=P^{0}$ for all $t>0$ and, from (6), $p_{i}^{*}=p_{i}^{0}$. In other words, model (3) has an infinite set of steady states $\boldsymbol{p}^{*}$ given by the solutions compatible with the set of conditions

$p_{i}^{*}=1-D-\alpha-\sum_{j \neq i} p_{j}^{*}, \quad i=1, \ldots, S$

When $P^{0} \neq 1-D-\alpha$, it follows from (3) that, for $D<1-\alpha$ and $P^{0}<P^{*}=1-D-\alpha\left(P^{0}>P^{*}\right)$, all fractions of occupied habitat $p_{i}(t)$ increase (decrease) monotonously to $p_{i}^{*}$ given by (6) as $t \rightarrow \infty$ since $P(t)<P^{*}\left(P(t)>P^{*}\right)$ for all $t>0$. Instead, for $D \geq$ $1-\alpha$, all fractions of occupied habitat tends uniformly to 0 as $t \rightarrow \infty$ and, hence, diversity collapse occurs.

It is important to mention that the existence of a non-trivial equilibrium of coexistence depends crucially on the assumption of the ecological equivalence in the way we have defined, i.e., as a linear trade-off function $\phi(c)$. We could have even defined a more strict version of ecological equivalence, as in Hubbell (2001), as an equivalence in per capita rates among all individuals of every species. Even such an strict ecological equivalence, as Hubbell (2001) states "permits complex ecological interactions among individ- uals so long as all individuals obey the same interaction rules". In particular, notice that under such a strict equivalence, our model predict as well a biodiversity collapse at the same threshold $D_{\mathrm{c}}=1-\alpha$, where $\alpha=m_{i} / c_{i}$.

Note that, when $\phi(c)$ is nonlinear, a model where species just colonize empty sites like ours (3) has no positive equilibrium solution different from the one with only one competitor, namely, the best competitor, i.e, that one with the lowest ratio $\phi\left(c_{i}\right) / c_{i}$. In this case, Eq. (3) do not support diversity. The underlying hierarchy that can be seen now as a ranking of species after colonization ability (the ratio $m_{i} / c_{i}$ ) leads to the extinction of all species but one, the best competitor. Once this point is reached the known critical threshold of habitat destruction for monospecific metapopulations remains true but now reads $D_{\mathrm{c}}=$ $1-\min _{i}\left\{\phi\left(c_{i}\right) / c_{i}\right\}$. For instance, if $\phi(c)=\alpha c^{2}$, the best competitor, i.e., the one which survives, will be the one with the lowest $c_{i}$. However, if $\phi(c)=\alpha \sqrt{c}$, then the survival will be the competitor with the highest $c_{i}$.

Although Eqs. (3) and (5) are nonlinear coupled equations and, so, it is not possible to solve them explicitly, a particularly relevant case can be analyzed using some approximations. Let us consider the linearized system

$\frac{\mathrm{d} p_{i}}{\mathrm{~d} t}=\sum_{j=1}^{S}\left(\frac{\partial f_{i}\left(\boldsymbol{p}^{*}\right)}{\partial p_{j}}\right) p_{j}, \quad i=1, \ldots, S$,

where the elements of the Jacobian matrix $L_{i j}=$ $\partial f_{i}\left(\boldsymbol{p}^{*}\right) / \partial p_{j}$ are given by:

$L_{i i}=c_{i}\left(1-D-\alpha-p_{i}^{*}-\sum_{j=1}^{S} p_{j}^{*}\right)$

(with $i=1, \ldots, S$ ) and, for $i \neq j$,

$L_{i j}=-c_{i} p_{i}^{*}$.

Now let us assume that all populations start at $t=0$ with the same, small values

$p_{i}(0)=\epsilon \gg 1-D-\alpha, \quad i=1, \ldots, S$.

Close to the fixed point $\boldsymbol{p}^{*}=(0, \ldots, 0)$, the linear approximation (8) reads (using the linear trade-off): $\mathrm{d} p_{i} / \mathrm{d} t=c_{i}(1-D-\alpha) p_{i}$ which has a solution: $p_{i}(t)=$ $\epsilon \exp \left(c_{i}[1-D-\alpha] t\right)$. 
At a given time step $T$, the probability of species $i$ under the linear approximation will be given by:

$P_{i}(T)=\frac{\mathrm{e}^{c_{i}[1-D-\alpha] T}}{\sum_{j=1}^{S} \mathrm{e}^{c_{j}[1-D-\alpha] T}}, \quad i=1, \ldots, S$.

Using the ordering $c_{i}=i / S$ and using that

$\sum_{j=1}^{S}\left(\mathrm{e}^{\gamma T / S}\right)^{j}=\frac{\mathrm{e}^{\gamma T / S}\left(1-\mathrm{e}^{\gamma T}\right)}{1-\mathrm{e}^{\gamma T / S}}$

where $\gamma=1-D-\alpha$, we obtain

$P_{i}(T)=Z \mathrm{e}^{(i-1) \gamma T / S}, \quad i=1, \ldots, S$.

Here $Z$ is the normalization factor:

$Z=\frac{1-\mathrm{e}^{\gamma T / S}}{1-\mathrm{e}^{\gamma T}}$

The last equations give a geometric distribution of abundances, in agreement with field observations from virgin habitats (Fisher et al., 1943; May, 1975) To restate our result in ecological terms: the process of ecological succession, i.e., the assembly of a competitive community from scratch $\left(p_{i}(0)=\epsilon \gg\right.$ $1-D-\alpha)$ onto an empty area under the rules of recruitment limitation and ecological equivalence gives rise to a geometric distribution of abundances. Alternatively, due to priority effects any other initial distribution of abundances will not ever tend to a the geometric distribution at equilibrium unless a strong perturbation resets the process of ecological succession again. Moreover, it is interesting to note that any geometric species rank distribution can be rewritten as a relative abundance distribution of the form $p(x) \approx x^{-\beta}$ with $\beta=1$. Thus, the same power-law scaling exponent has been obtained before in previous similar models where species compete for space in a somehow neutral way (Engen and Lande, 1996; Solé et al., 2000; McKane et al., 2000; Pueyo, 2003).

To finish, now we need to estimate a reasonable value $T^{*}$ in order to obtain some bound to the exact distribution. Since there is no obvious characteristic time scale to be used, we can estimate $T^{*}$ by using the condition

$\sum_{j=1}^{S} p_{j}\left(T^{*}\right)=1-D-\alpha$ i.e., the time at which the linear system reaches the maximum available population allowed by the nonlinear model. Strictly that equilibrium should be reached asymptotically as time tends to infinity. However, that time can be approximately computed using the properties of the geometric series. It can be easily shown that (16) can be written as

$\sum_{j=1}^{S} p_{j}\left(T^{*}\right)=\epsilon \frac{\mathrm{e}^{\gamma T^{*}}-1}{1-\mathrm{e}^{-\gamma T^{*} / S}}=1-D-\alpha$.

Hence, for $\gamma T^{*} / S$ close to zero and recalling that $\gamma=$ $1-D-\alpha$ it follows

$\frac{1}{T^{*}}\left(\mathrm{e}^{\gamma T^{*}}-1\right) \approx \frac{(1-D-\alpha)^{2}}{\epsilon S}$.

So, $T^{*}$ can be reasonably estimated from (17) as long as the number $S$ of species is large enough to guarantee that $\gamma T^{*} / S$ is close to zero. For instance, for $\alpha=0.5, S=150, \epsilon=10^{-3}$ and $D=0$, one obtains $T^{*}=4.129$ with $\gamma T^{*} / S=0.014$. In Fig. 1 we plot the exact, computed through the numerical integration of system (3) and the approximate (14) distributions. We can see an excellent agreement between them.

The behavior of this mean field model under habitat destruction is summarized in Fig. 2. Here we can see (Fig. 2a) that species extinctions start to occur close to $D_{\mathrm{c}}$ and that no extinction occurs below the threshold. Note that, according to Eq. (5), the characteristic time

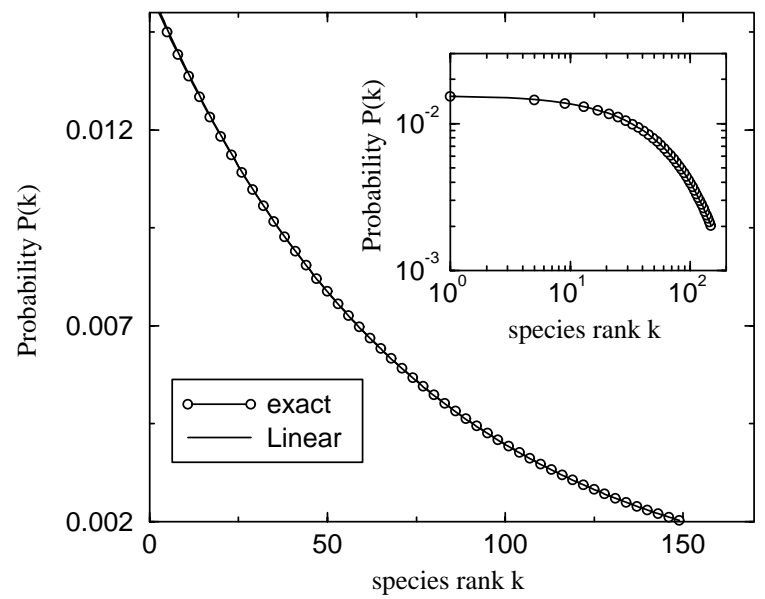

Fig. 1. Species-rank distribution from the mean field model (open circles) and from the linear approximation (line). A geometric series is obtained (showed in log-log scale in the inset). 


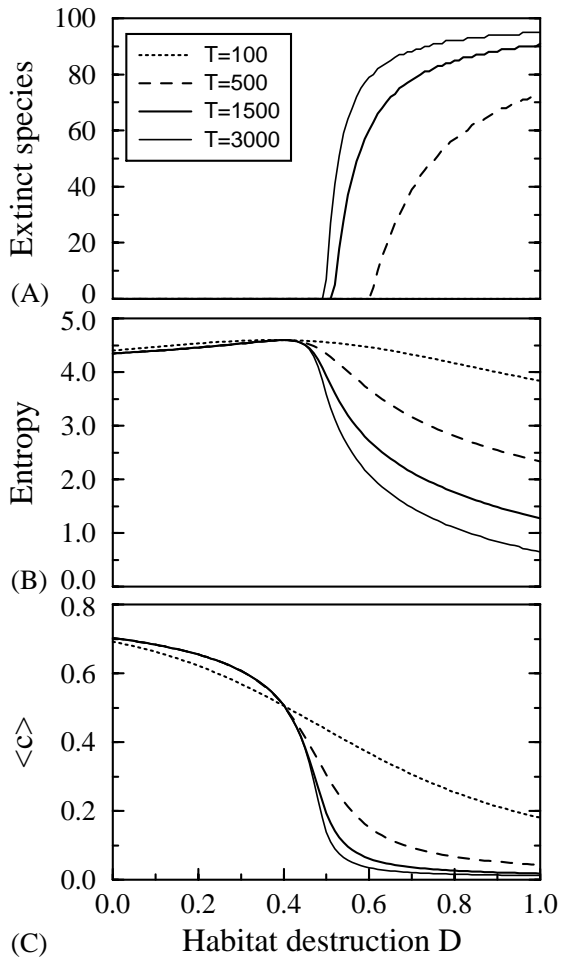

Fig. 2. Results obtained from the mean field model. Here we show: (A) the fraction extinct species; (B) the entropy, as defined by (15) and (C) the average colonization rate.

to extinction is propportional to $1 / m_{i}$, and, therefore, the loss of most persistent species can be very long. Once $D>D_{\mathrm{c}}$, however, extinctions sharply raise. A different metric is provided by the entropy defined as

$H(D)=-\sum_{j=1}^{S} P_{j}^{*} \log \left(P_{j}^{*}\right)$

where the stationary probabilities are:

$P_{j}^{*} \equiv \frac{p_{j}^{*}}{1-D-\alpha}, \quad j=1, \ldots, S$,

(see Fig. 2b). It first slightly increases (as the species occupying larger areas start to decrease in population and the system becomes more homogeneous) and then it rapidly falls off as species are lost. The average colonization shows a steady decrease for $D<D_{\mathrm{c}}$ but it also drops close to the threshold (Fig. 2c). Given that previous simulations have been done assembling the community from scratch, under the prescription given by (11), species that have higher colonization rates are occupying larger areas (see (14)). That is why they suffer first from habitat destruction. As in Tilman et al. (1994), habitat destruction affects first those species occupying larger areas. However, in contrast to previous models, the point to stress here is that a clearly defined common extinction threshold arises.

\section{Spatially-explicit model}

In the this section, we consider the introduction of spatially explicit effects, which are known to play a leading role (Dytham, 1994; Dythman, 1995; Bascomplte and Solé, 1996; Bascompte and Solé, 1998; Solé et al., 1996). Mean field models are often challenged by their spatially explicit counterparts. In particular, it has been shown that percolation processes can alter the presence and location of extinction thresholds (Bascomplte and Solé, 1996). Besides, finite-size effects will lead to a slow stochastic extinction of species unless immigration is introduced (Solé et al., 2000; McKane et al., 2000; Hubbell, 2001). The spatially explicit model is constructed using a stochastic cellular automaton (Durrett and Levin, 1994; Bascomplte and Solé, 1996) on a two-dimensional lattice of $L \times L$ discrete patches and periodic boundary conditions. Each site on the lattice can be either unoccupied or occupied by one of $S$ possible species. Actually the model can be accurately formalized as an interacting particle system as in Durrett (1999), where it is also included a multispecies neutral competition model. However, our model assumes local colonization of just neighboring sites as in Keymer et al. (2000). Moreover, although it has been stated that such neutral competition, where births are just possible on empty sites, precludes the system from stable species coexistence (Neuhauser, 1992), time to fixation of just one species can be very long when the system is large. In addition, external immigration prevents to reach monodominance and, provided low, could be added to our analysis without changing our conclusions - see also Hubbell (2001), Alonso (2004).

\subsection{Model definition}

Our system is modeled as a continuous-time Markov process where transitions occurs asynchronously 
so that within a short enough time interval only a single transition can take place. The state of the system at a time $t$ is described by giving the state of each site. The dynamics assumes two elemental processes:

1. Extinction. Any occupied site on the lattice can undergo extinction. Each species goes to extinction at a particular rate, $m_{i}=\alpha c_{i}$.

$j \stackrel{m_{i}}{\rightarrow} 0$.

2. Colonization. Any free site can undergo colonization.

$0 \stackrel{r_{k}}{\rightarrow} j$.

where $j$ is any potentially colonizing species from the neighborhood of the $k$ site. Colonization rates depend on the colonization pressure caused by neighboring occupied sites. Assuming that the $k$ site is free, colonization rate of this site at time $t$ can be computed as:

$r_{k}=\sum_{j=1}^{S} c_{j} \times \rho_{j}^{(k)}$

where $\rho_{j}^{(k)}$ is the local density, so the number of neighbors of the $k$ site that are occupied by species $j$ divided by the total number of neighboring sites, $z(z=4$, so von Neumann neighborhood was used in our simulations). Whenever a colonization event is to occur at that rate in $k$ site, the particular colonizing species is elected according to the probabilities given by the different non-zero terms in (22).

The particular simulation strategy is detailed elsewhere (Alonso and McKane, 2002). The algorithm is based on the rejection method described in Press et al. (1992). It is interesting to mention that by changing local density by global densities in (22), this formal model definition ensures the converge of averages values over replicas of stochastic simulations to the mean field patch occupancy model (Eq. (3)).

A first test of the interest of this model involves the analysis of the statistical regularities displayed by the virgin, non-destroyed habitat. A first observation is that after a decline in the number of species (which can be easily balanced by introducing immigration) the system settles down into a high-diversity state. A

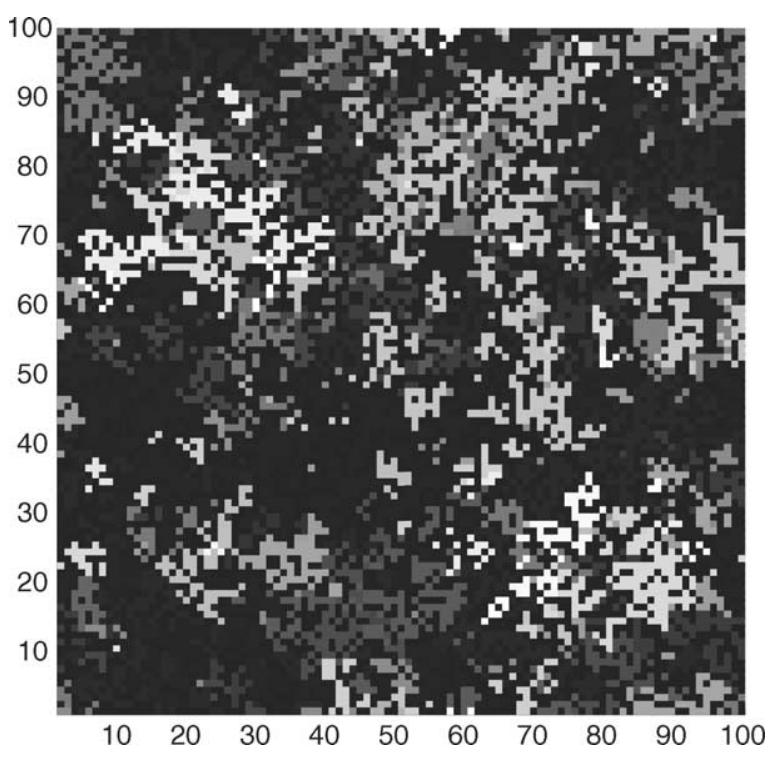

Fig. 3. Snapshot of the metapopulation model for $L=100$, $S=150$ and a linear trade-off with $\alpha=0.25$. Here the gray scale indicates the values of the colonization rate displayed by the specific species at each patch.

snapshot of a $L=100, S=150$ and $\alpha=0.25$ system is shown in Fig. 3. Here gray scales just indicate different species. We can see a patchy distribution of most species (some others have very scattered).

The patterns of species-abundance (consistent with the mean field model) and the species-area relations follow the observed patterns reported from field studies (Hubbell et al., 1999). These results are shown in Fig. 4, where the geometric distribution of species abundances is obvious from the species-rank distribution. The scaling law for the species-area relation is the same as the one observed in the Barro Colorado $50 \mathrm{Ha}$ plot (with an exponent $z=0.71 \pm 0.01$; see Hubbell et al. (1999), Brokaw and Busing (2000)). In summary, the imposed ecological equivalence through a simple trade-off together with the rules of local colonization are enough to recover two relevant, well-defined characteristics of real rainforests (compare with Hubbell et al. (1999)).

The effects of habitat destruction are, as expected from the mean field model, very important. In a previous study on habitat fragmentation in spatially-explicit models, it was shown that random destruction of patches leads to a critical point where sudden breaking 


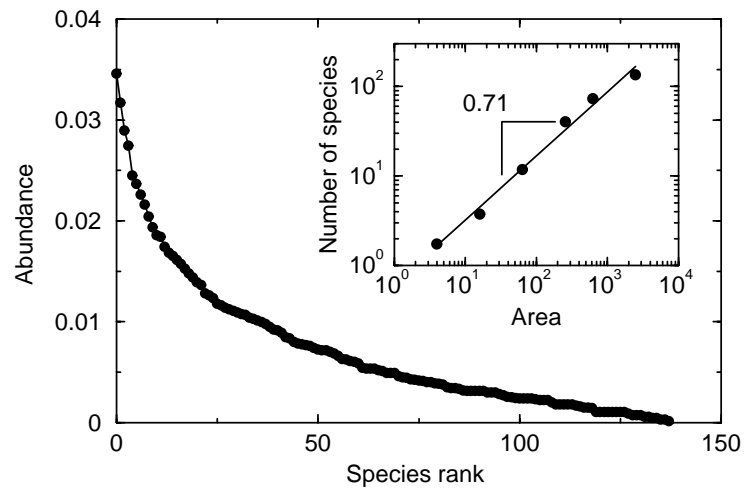

Fig. 4. Species-rank distribution in the spatially-explicit metapopulation model. A geometric distribution is obtained, as reported from field studies in rainforest plots. Inset: species-area curve for the same system; the exponent for the power law $S \propto A^{z}$ $(z=0.71)$ is indicated. into many small sub-areas promotes a rapid decay of the observed global population (Bascomplte and Solé, 1996; Bascompte and Solé, 1998; Solé and Bascompte, 2004). This occurs at the percolation value $D^{*} \approx 0.41$ (i.e. when the proportion of available sites is $1-D^{*} \approx 0.59$ ) where it can be shown that the largest available patch cannot longer be connected and splits into many sub-patches. The same effect has been found in our system. In Fig. 5(a), we show the biodiversity decay linked to the presence of a percolation phenomenon in the spatially-explicit landscape. As we can see, there is a steady decay in the number of species present as $D$ increases, with a rapid decay as $D^{*}$ is approached.

A different view of the decay can be obtained by plotting the Shannon entropy (Fig. 5(c)). We can see that it first increases as the best colonizers become less and less abundant (and the species-abundance distribution becomes more uniform). At $D^{*}$ the effective
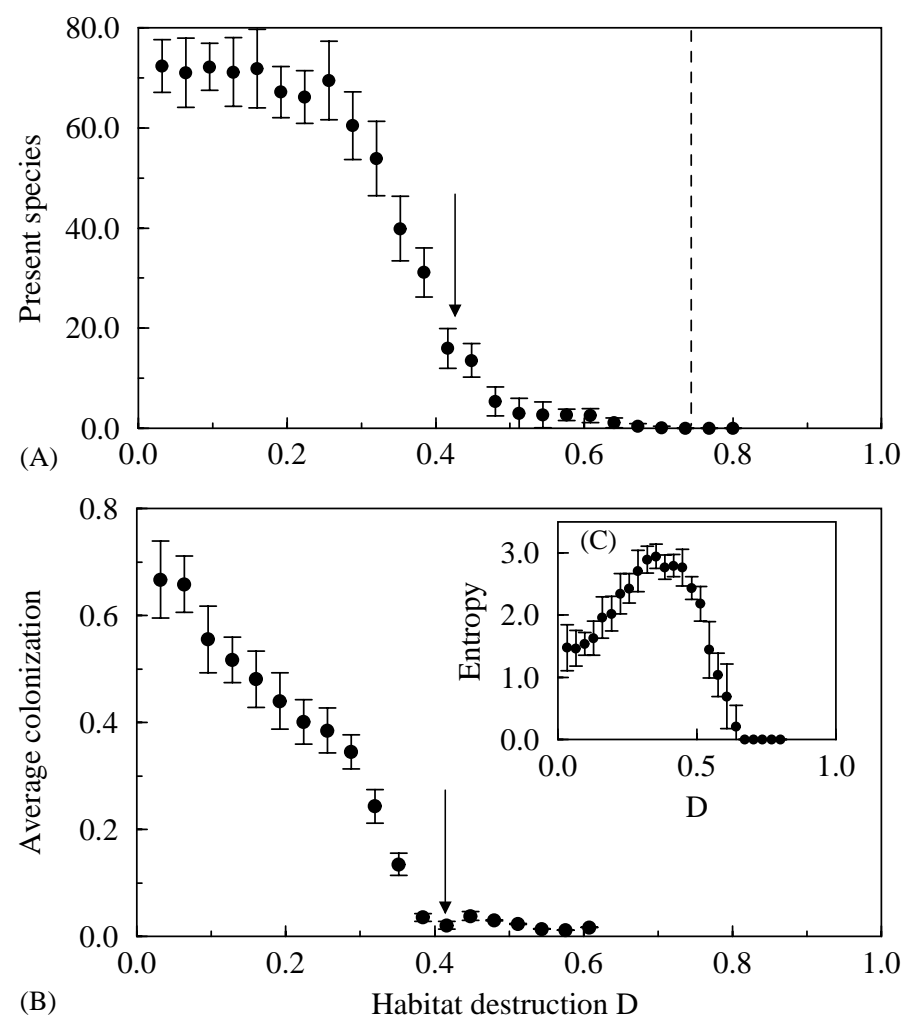

Fig. 5. Effects of habitat destruction on the spatial metapopulation model; here $L=50, S=150$ and $\alpha=0.25$. (A) number of species for different $D$ values; (B) average colonization rate: it shows a decay due to the extinction of best colonizators. In (C) the Shannon entropy is shown. The arrow indicates the percolation threshold $D^{*}=0.41$ and the dashed line the mean field threshold $D_{\mathrm{c}}=0.75$. 
loss of diversity reduces the value of the entropy which drops rapidly. As it was shown by the MF model, the average colonization rate also decreases (Fig. 5(b)). As $D^{*}$ is approached, the average colonization rate goes down. This indicates in fact that the rapid decrease in available habitat favours species with lower colonization rates in detriment to better dispersors but less persistent species.

\section{Discussion}

In this paper we have analyzed the properties of a simple model of metapopulation dynamics involving recruitment limitation and ecological equivalence. By recruitment limitation we meant that species can just colonize empty space. Instead of considering a hierarchical model of species competition, we have explored the consequences of recruitment limitation when habitat is destroyed and fragmented. Ecological equivalence is achieved by a simple trade-off where colonization is a linear function of extinction. The basic results obtained from our study are as given further.

1. The mean-field model of recruitment limitation introduces an infinite number of steady states (instead of a single attractor). This is an important factor in the path-dependence shown by the stochastic model. In particular and as a consequence of such path dependence, the system can be maintained far away from the expected geometric (or log-series) distributions unless strong perturbations undermine priority effects at least from time to time.

2. Under this scenario, the mean-field model predicts that biodiversity will experience a collapse close to the critical density $D_{\mathrm{c}}=1-\alpha, 0<\alpha<1$. This is very different from previous predictions, where extinction increases with $D$ in a nonlinear way but keeping surviving species at high $D$ values. Our result is closer to Stone's finding where trade-offs lead to rapid declines in diversity under small habitat loss.

3. The assumed ecological equivalence through the trade-off imposed by the colonization-extinction relation together with the random fluctuations implicit in the model rules lead to a stochastic multiplicative process with a geometric species-rank distribution of abundances, as predicted by theoret- ical models and to the right species-area relations reported from field studies in rainforests.

4. Under fragmentation, the system response is strongly influenced by percolation thresholds: once the critical value $D^{*}=0.41$ is reached, the fragmentation of the system into many patches leads to diversity collapse. The best colonizers are the first to get extinct. This model result should be qualified in the sense that real ecosystems are not isolated and, as a consequence, extinct good colonizers are most prone to be rescued by immigration from the region where the system is embedded.

A further comment to highlight is related to the geometric rank-abundance distribution we have obtained. It is formally equivalent to a power-low relative abundance distribution $p(x) \approx x^{-\beta}$, with $\beta=1$. This distribution has been derived under different approaches from sound first principles by making different assumptions. Tilman (1994) assumes it and finds the functional form of colonization rates that, by allowing coexistence, is compatible with it. He finds that the higher the competition rank a species has in the hierarchy, the lower its colonization rate is. Other similar trade-offs have also found to be responsible for geometric like distributions in competitive communities (Chave et al., 2002). Instead of trade-offs, Engen and Lande (1996), Solé et al. (2000), McKane et al. (2000), and Pueyo (2003) give the minimum requirements for geometric distribution to arise from simple dynamic population models. Pueyo (2003) even tests these predictions using an exceptional amount of data (almost a million of identified individual cells from phytoplankton samples) and finds high statistical significance for the scaling $p(x) \approx x^{-\beta}$ with $\beta=1$ within the abundance interval $10<n<10,000$. In particular, global constrains turn geometric distribution into the well-known log-series (Fisher et al., 1943), which is nothing but a power law with an exponential cut-off. In fact, Hubbell (2001) also finds the log-series distribution as limiting case of large systems at the metacommunity level. However, there is a common point of all these approaches: in order to get such scaling for the abundance distribution of species, species must be ecologically equivalent either explicitly assumed or trade-off mediated. Such ecological equivalence means equivalence in fitness, 
i.e., on average all species have the same probability to put one individual into the next generation. Natural selection and zero-sum dynamics (Hubbell, 2001), i.e, any increase in abundance of one species must be counterbalanced by an equivalent decrease in the abundance of other species, have poised and maintained species-rich communities in this critical state, where the scaling properties, such as those for the species-abundance distribution and the SAR, naturally arise (Solé et al., 2002; Pueyo, 2003).

Regarding habitat loss, our results must be re-analyzed using other approximations, such as other types of fragmentation involving correlations of different types (Dythman, 1995; Hill and Caswell, 1999), dynamic landscapes (Keymer et al., 2000) and screening effects (Alonso and Solé, 2000). In particular, there can be a non-trivial interaction between the predicted extinction threshold at $D=1-\alpha$, that should operate at global dispersion and the percolation well-known threshold at $D=0.41$, that would operate at strict first-neighbors local colonization. In natural conditions, colonization occurs neither completely globally nor completely locally. In any case, our results seem to be robust and make the point of a common extinction threshold for all species as habitat is lost resulting in a biodiversity collapse.

Even though geometric rank-abundance distributions seem to hold provided that ecological equivalence is guaranteed, its particular expression can have strong consequences in the way habitat destruction affects diversity. In contrast to other previous models (Tilman et al., 1994; Stone, 1995), our work predicts the existence of a common destruction threshold. So, rather in the line of Stone's findings, it predicts a devastating high non-linear effect of small amounts of habitat losses on community integrity. Different departures from our model including contact-like processes, voter models, and external immigration fully confirm this result (Alonso, 2004). The fact that chance events and limited recruitment can slow down competitive exclusion and lead to strong path-dependence makes our present results likely to apply to a wide range of current scenarios of rapid diversity loss. Our model should provide a better understanding of how competitive communities are organized and how fragile they are when habitat loss takes place.

\section{Acknowledgements}

RVS and DA thank the members of the CSL for useful discussions. DA would like to thank the MACSIN research group at the UFMG, Belo Horizonte, Brasil for providing constant support and a nice environment to work. This work has been supported by grants CICYT BFM 2001-2154 (RVS), CIRIT FI00524 (DA), BFM2002-04613-C03-03 (JS), and by the Santa Fe Institute.

\section{References}

Alonso, D., 2004. The Stochastic Nature of Ecological Interactions: Communities, Metapopulations, and Epidemies. Ph.D. thesis, Polytechnic University of Catalonia.

Alonso, D., McKane, A., 2002. Extinction dynamics in mainlandisland metapopulations: An $n$-patch stochastic model. Bull. Math. Biol. 64, 913-958.

Alonso, D., Solé, R.V., 2000. The divgame simulator: a stochastic celular automata of rainforest dynamics. Ecological. Modelling. 133, 31-141.

Barkai, A., McQuaid, C., 1988. Predator-prey role reversal in a marine benthic ecosystem. Science 242, 26-64.

Bascomplte, J., Solé, R.V., 1996. Habitat fragmentation and extinction thresholds in spatially-explicit models. J. Anim. Ecol. 65, 465-473.

Bascompte, J., Solé, R.V., 1998. Effects of habitat destruction in a prey-predator metapopulation model. J. Theor. Biol. 195, 383-393.

Brokaw, N., Busing, R.T., 2000. Niche versus chance and tree diversity in forest gaps. TREE 133, 133-141.

Chave, J., Muller-Landau, H.C., Levin, S.A., 2002. Comparing classical community models: theoretical consequences for patterns of diversity. Am. Nat. 159, 1-23.

Crowley, P.H., McLetchie, D.N., 2002. Trade-offs and spatial life-history strategies in classical metapopulations. Am. Nat. 159, 190-201.

Durrett, R., 1999. Stochastic spatial models. SIAM Review 41, $677-718$.

Durrett, R., Levin, S., 1994. The importance of being discrete (and spatial). Theor. Pop. Biol. 46, 363-394.

Dytham, C., 1994. Habitat destruction and competitive coexixtence: a cellular model. J. Anim. Ecol. 63, 490-495.

Dythman, C., 1995. The effect of habitat destruction pattern on species persistence: a cellular model. OIKOS 74, 340-344.

Ehrlich, P., Ehrlich, A., 1981. Extinction. Oxford University Press, Oxford.

Engen, S., Lande, R., 1996. Population dynamics models generating species-abundance distributions of the gamma type. J. Theor. Biol. 178, 331-355.

Fisher, R., Corbet, A., Williams, C., 1943. A theoretical distribution for the apparence abundance of different species. J. Anim. Ecol. 12, 42-58. 
Fisher, R., Corbet, A., Williams, C., 1951. Copepodology for the ornitology. Ecology 32, 571-577.

Hanski, I., 1999. Metapopulation Ecology. Oxford University Press, Oxford.

Hill, M., Caswell, H., 1999. Habitat fragmentation and extinction threshold on fractal landscapes. Ecol. Lett. 2, 121-144.

Hubbell, S.P., 2001. The Unified Neutral Theory of Biodiversity and Biogeography. Princeton University Press, Princeton, NJ.

Hubbell, S.P., Foster, R.B., O’Brien, S.T., Harms, K.E., Condit, R., Wechsler, B., Wright, S.J., de Lao, S.L., 1999. Light gap disturbances, recruitment limitation, and tree diversity in a neotropical forest. Science 283, 554-557.

Kareiva, P., Wennengren, U., 1995. Connecting landscape patterns to ecosystem and population process. Nature 373, 299302.

Keymer, J.E., Marquet, P.A., Velasco-Hernàndez, J.X., Levin, S.A., 2000. Extinction thresholds and metapopulation persistence in dynamic landscapes. Am. Nat. 156, 478-494.

Leakey, R., Lewin, R., 1995. The Sixth Extinction. Dubbleday, New York.

Lehman, C.L., Tilman, D., 1997. Competition in spatial habitats. In: Tilman, D., Kareiba, P. (Eds.), Spatial Ecology: the Role of Space in Population Dynamcis and Interspecifics Interactions, vol. 30 (Monographs in Population Biology). Princeton University Press, Princeton, NJ, pp. 85-133.

Levin, S.A., 1999. Fragile Dominion. Perseus Books, Reading, MA.

Levins, R., 1969. Some demographic and genetic consequences of environmental heterogeneity for biological control. Bull. Entomol. Soc. Am. 15, 227-240.

MacArthur, R.H., Wilson, E.O., 1967. The theory of Island Biogeography. Princeton University Press, Princeton, NJ.

May, R.M., 1975. Patterns of species-abundances and diversity. In: Cody, M.L., Diamond, J.M. (Eds.), Ecology and Evolution of Communities. pp. 81-118.

McKane, A., Alonso, D., Solé, R.V., 2000. A mean field stochastic theory for species rich assembled communities. Phys. Rev. E. $62,8466-8484$.
Neuhauser, C., 1992. Ergodic theorems for the multy-type contact process. Prob. Theor. Rel. Fields 91, 467-506.

Pimm, S.L., 1991. The Balance of Nature? The University of Chicago Press, Chicago.

Pimm, S.L., Russell, G.J., Gittleman, J.L., Brooks, T.M., 1998. The future of biodiversity. Adv. Complex Sys. 1, 203.

Press, W.H., Teukolsky, S.A., Vetterling, W.T., Flannery, B.P., 1992. Numerical Recipes in C. Cambridge University Press, Cambridge.

Pueyo, S., (2003). Irreversibility and Criticality in the Biosphere. Ph.D. thesis, University of Barcelona.

Skellam, J.G., (1951). Random dispersal in theoretical population. Biometrika 38, 196-218.

Solé, R., Manrubia, S., Bascompte, J., Delgado, J., Luque, B., 1996. Phase transitions and complex systems. Complexity 1 , $13-18$.

Solé, R.V., Alonso, D., McKane, A., 2000. Scaling in a network model of multispecies communities. Physica A 286, 337-344.

Solé, R.V., Alonso, D., McKane, A., 2002. Self-organized instability in complex ecosystems. Phil. Trans. R. Soc. London. ser. B 357, 667-681.

Solé, R.V., Bascompte, J., 2004. Complexity and Self-organization in Evolutionary Ecology. Princeton University Press, Princeton, NJ.

Stone, L., 1995. Biodiversty and habitat destruction: a comparative study of model forest and coral reefs systems. Proc. R. Soc. London B 262, 381-388.

Tilman, D., 1982. Resource Competition and Community Structure. Princeton University Press, Princeton, NJ.

Tilman, D., 1994. Competition and biodiversity in spatially structured habitats. Ecology 75, 2-16.

Tilman, D., 1999. Diversity by default. Science 283, 495-496.

Tilman, D., Kareiva, P. (Eds.) 1997. Spatial Ecology. The Role of Space in Population Dynamics and Interspecific interactions, vol. 30 (Monographs in Population Biology). Princeton University Press, Princeton, NJ.

Tilman, D., May, R.M., Lehman, C.L., Novak, M.A., 1994. Habitat destruction and extinction debt. Nature 371, 65-66. 